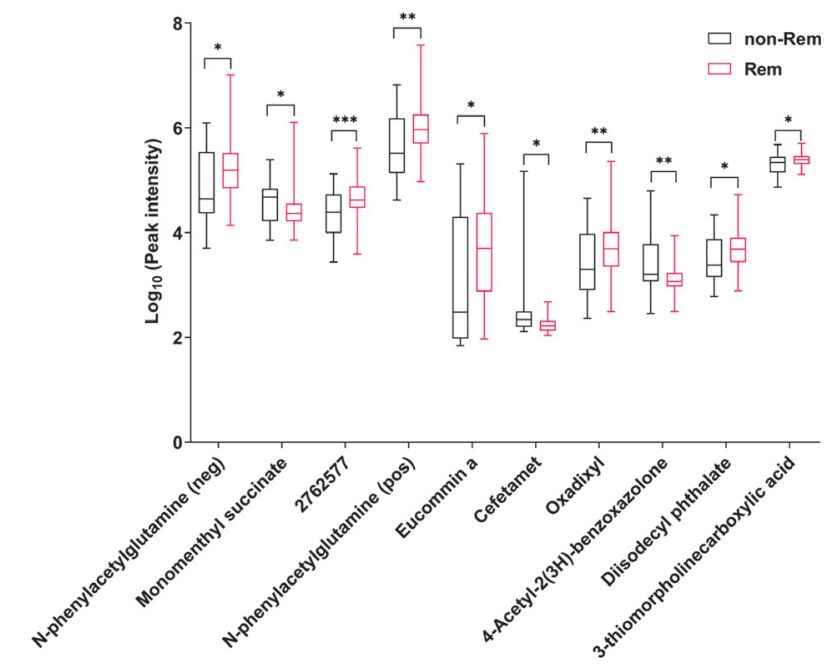

Abstract IDDF2021-ABS-0091 Figure 4

predictive performance of FMT efficacy in CD. The combination of them will better serve the clinical decision-making. ClinicalTrials.gov: NCT1793831.

\section{IDDF2021-ABS-0093 ANALYSIS OF THALIDOMIDE ASSOCIATED PERIPHERAL NEUROPATHY IN PATIENTS WITH CROHN'S DISEASE}

Xiang Peng*, Wei Chen, Jiayin Yao, Jun Deng. Department of Gastroenterology, Guangdong Provincial Key Laboratory of Colorectal and Pelvic Floor Disease, The Sixth Affiliated Hospital of Sun Yat-Sen University, China

\subsection{6/gutjnl-2021-IDDF.136}

Background Crohn's disease (CD) is an incurable chronic nonspecific inflammatory bowel disease associated with environmental, genetic, infectious and immune factors. A previous study has shown that thalidomide alleviates symptoms and signs of children and adolescents with CD. But it is limited by thalidomide-induced peripheral neuropathy.

Methods Recruited were patients paying consecutive visits for refractory and active CD at the Sixth Affiliated Hospital, Sun Yat-sen University, between August 2016 and November 2021. We evaluated the peripheral nerve symptoms and EMG.

Results 69 patients were recruited in this study; among them, 31 patients had peripheral neuropathy symptoms and 24 were EMG positive. But of the 38 asymptomatic patients, 20 remained EMG positive. Of these 44 patients with peripheral neuropathy, 30 had mild impairment, 13 had moderate impairment, and one had severe impairment. 42 patients had sensory neuropathy, 1 patient had motor neuropathy, and 1 patient had both

Peripheral neuropathy-free patients were found in 17 of 52 patients who received thalidomide for 1 year. After discontinuation of thalidomide, $25.53 \%$ had complete response, $53.19 \%$ had partial response and $21.28 \%$ had no response. About 3.91 months later, peripheral neurological symptoms began to show relief.

Conclusions Peripheral neuropathy is common in patients with inflammatory bowel disease, but is generally mild and tolerable, with predominantly sensory nerve damage. Peripheral neuropathy should be monitored by electromyography during thalidomide administration.

\section{IDDF2021-ABS-0100 PRIORITIZATION ON WHOLE-SLIDE IMAGES OF CLINICAL GASTRIC CARCINOMA BIOPSIES THROUGH A WEAKLY SUPERVISED AND ANNOTATION-FREE SYSTEM}

${ }^{1}$ Alex Ngai Nick Wong*, ${ }^{1}$ Martin Ho Yin Yeung, ${ }^{2}$ Cheong Kin Ronald Chan, ${ }^{2}$ Angela Zaneta Chan, ${ }^{1}$ Chun Yin Wong, ${ }^{2}$ Tsz Yan Joyce Chan, ${ }^{1} J u n g$ Sun Yoo, ${ }^{1}$ Chi Ming Wong. ${ }^{1}$ Department of Health Technology and Informatics, The Hong Kong Polytechnic University, Hong Kong; '2Department of Anatomical and Cellular Pathology, The Chinese University of Hong Kong, Hong Kong

\subsection{6/gutjnl-2021-IDDF.137}

Background Recent advances in digital pathology and artificial intelligence provided the potential for the development of robust clinical diagnostic tools to support pathologists. With a worldwide shortage of pathologists and increased cancer screening programs, digital pathology is expected to grow and will revolutionize the workflow of pathologists. However, the development is hindered by the time-consuming whole slide imaging (WSI) annotation process during model training and lack of experience in integration into clinical workflow. This study aims to target these issues by developing a weakly supervised and annotation-free system to triage suspicious gastric carcinoma biopsies.

Methods We digitized 965 (2795 images) gastric biopsy cases from Prince of Wales Hospital, including 648 (2062 images) ,192 (576 images) and 125 (157 images) cases for model development and as first and second external validation sets respectively to evaluate model performance. A neural network-based binary classifier for cancer detection was trained using 41 averaged cellular features generated from WSI and case diagnostic labels. Accuracy and AUC were measured for model performance. To evaluate our model to carcinoma case prioritization, another 90 cases (122 images) were digitized. 16 pathologists from 4 centers were recruited for a randomized study to review these cases in a simulated clinical workflow, with and without the assistance of our system. The final diagnosis was compared to ground truth and time spend per case was measured to demonstrate the clinical utility of triage.

Results We developed a weakly supervised system (GastroFLOW), achieving the accuracy of $78-83 \%$ and AUC of 0.92 0.95 on external validation sets, which can skip $69-78 \%$ of the non-carcinoma cases while retaining $100 \%$ sensitivity. In a simulated pathology clinical setting, GastroFLOW prioritized all CA cases forward. Moreover, 52.29-54.79\% time was saved from viewing all non-carcinoma cases as compared with random scenarios (IDDF2021-ABS-0100 Figure 1).

Conclusions In summary, we developed a clinical decision support tool from annotation-free WSI, based on automatically generated cellular features for gastric carcinoma. GastroFLOW
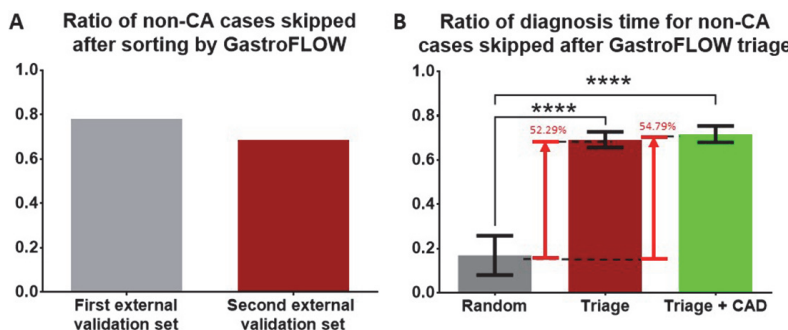

Abstract IDDF2021-ABS-0100 Figure 1 
allows optimization of daily pathologist workload to meet case turn-around-time promptly for urgent cases, improving cancer patient management, paving the way for the application of computational pathology in the workflow of a histopathology laboratory.

\section{IDDF2021-ABS-0105 PREVALENCE OF 'POUCH FAILURE' OF THE ILEOANAL POUCH IN ULCERATIVE COLITIS: A SYSTEMATIC REVIEW AND META- ANALYSIS}

'Zaid Alsafi*, ${ }^{2}$ Alice Snell, ${ }^{3}$ Jonathan P Segal. ${ }^{1}$ Imperial College London, UK; ${ }^{2}$ Department of Gastroenterology, Northwick Park Hospital, London, UK; ${ }^{3}$ Department of Gastroenterology, Imperial College Healthcare NHS Trust, London, UK

\subsection{6/gutjn-2021-IDDF.138}

Background The Ileoanal pouch (IPAA) provides patients with ulcerative colitis (UC) that has not responded to medical therapy an option to retain bowel continuity and defecate without the need for a long-term stoma. Despite good functional outcomes, some pouches fail, requiring permanent diversion, pouchectomy or a redo pouch. The incidence of pouch failure ranges between $1 \%-15 \%$ in the literature. Understanding the incidence of pouch failure is vital in counselling patients considering this operation. Therefore, we conducted a systematic review and meta-analysis aiming to define the prevalence of pouch failure in patients with UC who have undergone IPAA using population-based studies.

Methods We searched Embase, Embase classic and PubMed from 1978- 31st of May 2021 to identify cross-sectional studies that reported the prevalence of pouch failure in adults ( $\geq 18$ years of age) who underwent IPAA for UC. We handsearched the references from eligible studies and the proceedings from Inflammatory bowel disease conferences. We combined the proportion of patients with pouch failure in each study to give a pooled prevalence for each study. We performed a random-effects model in order to pool the data to provide an estimate of the prevalence of pouch failure. Heterogeneity was assessed using the $\mathrm{I}^{2}$ statistic.

Results Thirty-three studies comprising 29,153 patients were analysed. After a five-year follow-up period, the prevalence of pouch failure was 4\% (95\%CI 2\%-6\%) (IDDF2021-ABS-0105 Figure 1). Between 5-10 years of follow-up, the prevalence was 4\% (95\%CI 3\%-7\%) (IDDF2021-ABS-0105 Figure 2). This increased to $11 \%(95 \% \mathrm{CI} 7 \%-16 \%)$ with over 10 years

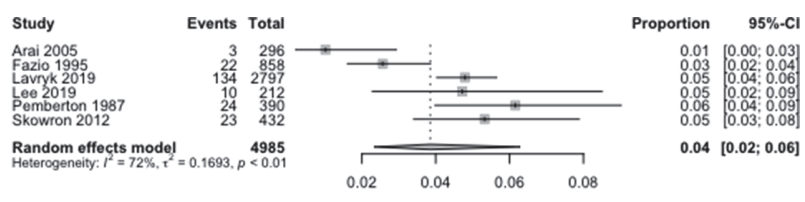

Abstract IDDF2021-ABS-0105 Figure 1

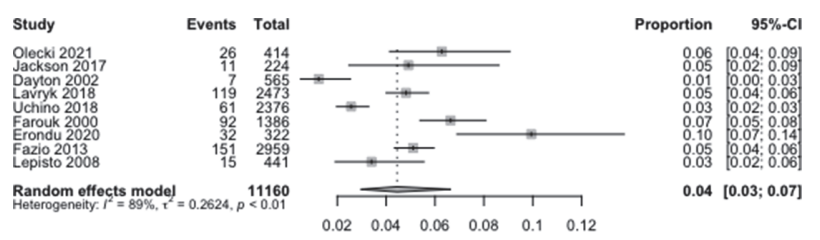

Abstract IDDF2021-ABS-0105 Figure 2

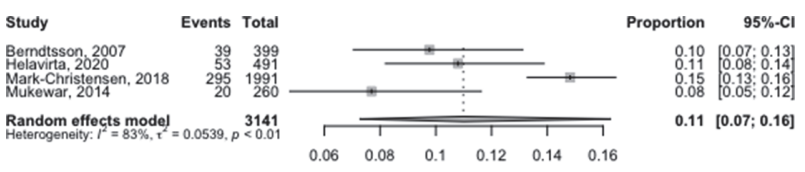

Abstract IDDF2021-ABS-0105 Figure 3

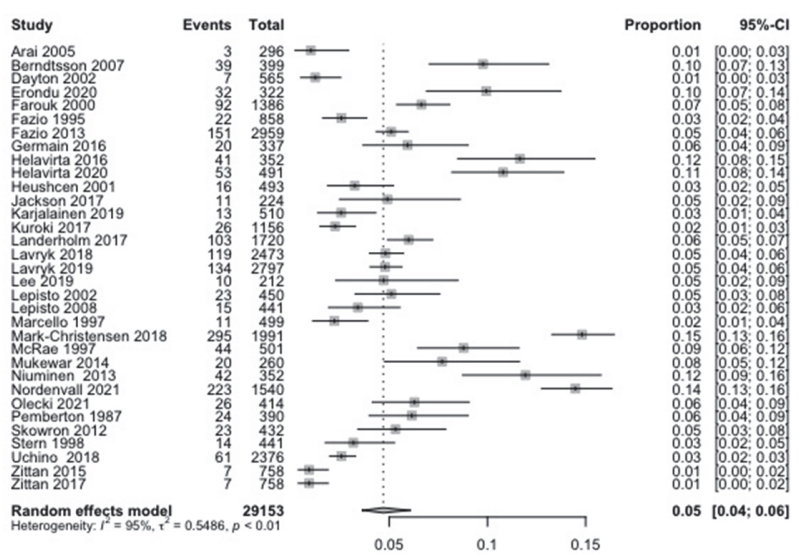

Abstract IDDF2021-ABS-0105 Figure 4

of follow-up (IDDF2021-ABS-0105 Figure 3). The overall prevalence of pouch failure was $5 \%$ (95\%CI 4\%-6\%) (IDDF2021-ABS-0105 Figure 4).

Conclusions The prevalence of pouch failure in patients over the age of 18 who have undergone restorative proctocolectomy in UC is $11 \%$ after a 10 -year follow-up. These data are important for counselling patients considering this operation. Importantly, for those patients with UC being considered for a pouch, their disease course has often resulted in both physical and psychological morbidity and hence providing accurate expectations for these patients is vital.

\section{IDDF2021-ABS-0109 OBG-LIKE ATPASE 1 (OLA1) CONTRIBUTES TO GASTRIC CANCER PROGRESSION AND CAN BE A NOVEL THERAPEUTIC TARGET}

${ }^{1}$ Wen Cai*, ${ }^{1}$ Siyuan Xie, ${ }^{2}$ Weiting Ge, ${ }^{3}$ Hanguang Hu, ${ }^{1}$ Jianshan Mao, ${ }^{2}$ Jiawei Zhang. 'Department of Gastroenterology, Second Affiliated Hospital of Zhejiang University School of Medicine, China; ${ }^{2}$ Cancer Institute (Key Laboratory of Cancer Prevention and Intervention, China National Ministry of Education), the Second Affiliated Hospital, School of Medicine, Zhejiang University, China; ${ }^{3}$ Department of Medical Oncology, the Second Affiliated Hospital, School of Medicine, Zhejiang University, China

\subsection{6/gutjpl-2021-IDDF.139}

Background Obg-like ATPase 1(OLA1) is a member of the GTPase protein family but utilizes ATP over GTP in humans. OLA1 has been reported to be involved in lung and breast cancer progression, however, it remains unclear whether OLA1 plays a critical role in gastric cancer.

Methods TCGA and GEO datasets data were collected to identify differential gene expression profiles in gastric cancer patient samples. Western blot and real-time polymerase chain reaction were performed to evaluate OLA1 expression in gastric cancer cells and normal gastric epithelium. Cell count Kit8, Propidium iodide staining assay, colony formation assay, wound-healing and transwell assays were applied to analyze the effects of OLA1 on gastric cancer cell proliferation and metastasis in vitro. Moreover, the Subcutaneous gastric cancer 